\title{
II processo tutoriale nell'ambito della formazione del Tecnico Sanitario di Laboratorio Biomedico neoassunto nel Laboratorio di Microbiologia
}

\section{Iole Caola, Cira Nuzzo}

Laboratorio di Microbiologia e Virologia - Ospedale S. Chiara - Trento

Key words: Tutorship, mentor, activity plan, laboratory technician

\section{SUMMARY}

The tutorship in the training of the new engaged Biomedical Technician in the Microbiology Laboratory

The training period for a new engaged laboratory technician in the microbiological laboratory is considered in this work. Experience and reflective practice are the key elements in order to develope professional skills and new knowledge in adults.

Learning occurs through the interaction between persons and their concrete experience.

The main subject, acting as a training developer, is the mentor: he/she is the expert colleague that create an ad personam relationship with the neophyta, to support the development of professionals skills and to psychologically sustain the new member in such a difficult period. The high complexity of the laboratory framework requires an intense and articulated tutorship, in order to link the learning times of the new technician with the working times and priorities, managing the learning incentives by a careful planning.

In the tutorial function is particulary important the use of methods that facilitate the experential learning reflective practice, e.g. contract, briefing, practice and debriefing.

In the working agreement the needs are taked in account, the training objectives and the time for the final skills are defined. The main operating instrument is the training plan, where the specific goals are divided in professional activities that will enable the acquisition of the final skills in a well defined working period, also taking into account a specific progressive control. Regarding the specific framework of the microbiology lab, eight points have been identified in the training plan to reach the main professional skills, while the activities, that are necessary to reach the desired results in a specific time period, have been defined.

\section{INTRODUZIONE}

Questo lavoro propone un percorso di inserimento nell'attività lavorativa dei servizi di laboratorio di un tecnico sanitario di laboratorio biomedico (TSLB) neoassunto, individuando in questo particolare momento della carriera professionale la necessità di un'azione formativa finalizzata allo sviluppo, crescita, autonomia e autorealizzazione dello stesso nell'ambito non solo professionale ma anche personale.

Il nuovo assunto, in quanto adulto, deve poter esplorare ed affrontare i suoi specifici bisogni formativi all'interno di un progetto articolato e flessibile, dove l'apprendimento si arricchisce con 1'attivazione di una significativa riflessione sull'esperienza, come modalità privilegiata di acquisizione del sapere "tacito", caratteristico anche della dimensione tecnica, intesa come expertise applicata allo specifico contesto del laboratorio.

Il soggetto in formazione si inserisce in una organizzazione, nel cui interno viene promosso un processo di esplicitazione, creazione e circolazione di sapere, tradotto in concrete pratiche operative, che crea le condizioni per presidiare meglio le attività di lavoro, diffondere e scambiare le cono- scenze personali per renderle patrimonio dell'organizzazione, contribuendo al miglioramento continuo dei processi.

La figura da affiancare al neoassunto per attivare, presidiare e facilitare i processi di apprendimento e di autovalutazione all'interno di questo contesto organizzativo è quella di un tutor (mentore), che attraverso una relazione duale tra "individuo in formazione" ed esperto porta ad uno sviluppo di competenze professionali con un sostegno psicologico importante in un momento così impegnativo della carriera.

La microbiologia, ed in particolare la batteriologia, è una delle aree di laboratorio di maggiore complessità operativa ed interpretativa. La "risorsa uomo" è fondamentale: è necessaria la presenza di personale tecnico esperto, specializzato e motivato per garantire la qualità delle prestazioni. Per questo motivo sono stati elaborati un percorso tipo e uno strumento, "il piano di inserimento" che possa fungere da stimolo per trasformare la fase di inserimento del neoassunto da un processo informale e spontaneo ad un percorso programmato, riconosciuto e formalizzato, sostenuto anche da un training formativo rivolto a coloro 
che diventeranno tutor / mentor / coach .

Lo sviluppo delle competenze nelle organizzazioni: l'importanza dell'inserimento lavorativo La sempre maggiore complessità dell'organizzazione lavorativa in ambito sanitario richiede professionisti con elevata capacità progettuale, di scelta e di problem solving, piuttosto che di mera applicazione di regole e procedure. Quando un individuo esce dalla sede formativa deve confrontarsi con esigenze lavorative reali che richiedono una risposta professionale competente (8) e basata non solo sulla formazione disciplinare ricevuta, ma soprattutto dalla capacità di mettere in atto strategie efficaci di soluzione ai problemi emergenti. Tale risposta appare sempre più il frutto di un interscambio a livello di equipe. Il neoassunto, in quanto membro di un gruppo operativo, ricostruisce progressivamente una propria configurazione professionale effettiva e una concreta competenza lavorativa sulla base di episodi concreti che vengono affrontati, interpretati, raccontati e discussi dagli attori che ne sono coinvolti. Il risultato finale non è trasmissibile ad altri esclusivamente tramite un linguaggio formalizzato ed in modo diretto.

Ciò ha evidenti implicazioni sulla identificazione delle abilità di base che andrebbero attivate dal neoassunto, sui suoi tempi di apprendimento e sulla esigenza di garantire spazi di riflessione sull'esperienza diretta.

Nella attuale situazione di profonda trasformazione strutturale ed amministrativa dei servizi sanitari, vengono valorizzati i modelli organizzativi finalizzati ad un "miglioramento continuo della Qualità", nonché all'efficacia dei servizi erogati: tutto ciò presuppone sia una presenza attiva dei professionisti stessi, sia un coinvolgimento personale assai elevato nella gestione delle attività di lavoro.

Nel periodo di inserimento del neoassunto si concretizza una funzione di "messa alla prova", con dosi crescenti di autonomia individuale, di capacità operative e procedurali e di abilità di integrazione delle conoscenze possedute; questo con la finalità di individuare, in una relazione di tipo tutoriale attuata con il professionista esperto, le soluzioni più soddisfacenti ai problemi professionali che si presentano in una concreta situazione di lavoro.

$\mathrm{Al}$ processo tutoriale non compete la valutazione di tipo certificativo di idoneità del periodo di prova, ma esclusivamente una valutazione di tipo formativo. Il percorso comunque non potrà non tenere costantemente presente l'obiettivo di formare sulle funzioni, compiti e attività che saranno oggetto di valutazione, come indicato dalla nor- mativa, dopo sei mesi di prova. La responsabilità di questa valutazione compete al Coordinatore TSLB e al Responsabile di settore mentre il giudizio di idoneità, al direttore di U.O. (3).

\section{La funzione di tutorship: \\ finalità, competenze e declinazioni}

Le funzioni di tutoring rivolte al neoassunto TSLB sono affidate al professionista esperto che, nell'ambito della sua attività di servizio, svolge funzioni di guida e facilitazione all'apprendimento.

La tutorship assume il significato di sostegno a uno sforzo di apprendimento dall'esperienza, a un impegno di coinvolgimento personale in un percorso che richiede riflessività costante, capacità di progettare, scegliere, agire in un contesto di problem solving piuttosto che semplice imitazione di modelli professionali e uso di abilità acquisite meccanicamente (7).

Nei contesti organizzativi il mentore, con una relazione ad personam supporta il neoassunto in un momento tanto impegnativo del percorso professionale (4).

La relazione tra esperto e novizio prosegue fino a quando quest'ultimo non ha sviluppato pienamente le proprie potenzialità.

Nel reale svolgimento della funzione di tutorship sono implicate due aree di competenza:

- competenze professionali specifiche: riferite non solo a quelle previste dal profilo professionale di appartenenza, ma anche a quelle declinate ed agite nello specifico contesto (tipo di servizio)

- competenze trasversali sui processi cognitivi, relazionali, organizzativi e di apprendimento, "nucleo centrale" della funzione tutoriale.

Le attività si ascrivono alle aree di analisi dei bisogni, alla progettazione dell'intervento formativo, alla programmazione operativa, alla conduzione e gestione delle attività previste ed infine alla valutazione dei risultati.

Vista la complessità della tutorship, è necessario progettare, promuovere ed implementare un programma formale specifico, nonché definire un percorso formativo indirizzato alle figure deputate a svolgere tale funzione (5). La tutorship all'interno dei servizi di laboratorio non si deve configurare come un processo informale e spontaneo, come di fatto è nella maggioranza delle organizzazioni (i senior trasferiscono occasionalmente cultura, competenze ed esperienze ai junior), ma come un percorso formalizzato e gestito.

Il tutor deve riconoscere ed aiutare il nuovo assunto sia a superare le diverse difficoltà incontrate nel suo inserimento nel contesto lavorativo, sia ad affrontare gli eventuali contrasti tra esigen- 
ze lavorative e bisogni formativi (1)

Il tutor gestisce, in itinere e relazionandosi con altre figure professionali, diversi e notevoli aspetti della fase di formazione, coinvolgendo anche il neoassunto. In particolare: a) verifica il livello di apprendimento, individuando le strategie che si sono rivelate più efficaci; b) procede ad eventuali integrazioni o correzioni, evidenziando le difficoltà incontrate al fine di adottare adeguate misure per superarle, ponendo particolare attenzione a non minare l'autostima del neofita; c) verifica il tipo di relazione, individuale e di gruppo, instaurata dal neoassunto ed i suoi effetti sul piano dell'apprendimento; d) aiuta a valutare gli effetti dell'inserimento sull'organizzazione del servizio, in modo da suggerire possibili miglioramenti per le future nuove assunzioni. Il tutor, in definitiva, è parte integrante del progetto e del processo di formazione, inclusa la valutazione critica delle proprie performance. Nella fase di valutazione dei risultati va coinvolto il neoassunto, non solo con l'intento di riflettere sul suo apprendimento, ma anche per introdurre nella persona una vera attenzione critica sul "senso" di quello che fa ogni giorno, premessa necessaria e spinta potente per incamminarsi sulla strada della qualità.

\section{Percorso "tipo" standard di inserimento di un TSLB neoassunto nel laboratorio di microbio- logia, settore batteriologia}

L'attività svolta nel Laboratorio di Microbiologia, ed in particolare nel settore batteriologia, è caratterizzata dall'uso prevalente di "tecniche convenzionali" (esame microscopico, colturale, isolamento, ecc.) che richiedono personale tecnico esperto, specializzato e qualificato e che necessita di un lungo periodo di apprendimento e tirocinio (9).

Il setting formativo nei servizi di laboratorio si presenta con caratteristiche molto diverse da un contesto specifico all'altro, in relazione alla presenza di attività programmate o urgenti, all'apertura diurna o continuativa sulle 24 ore del servizio, alla presenza di equipe multiprofessionali, agli orari e al numero di operatori coinvolti nella tutorship, ai carichi di lavoro, all'organizzazione più o meno settorializzata del lavoro.

In tali contesti ad elevata complessità lavorativa va attuata una forma di tutorship ancora più articolata ed intensa per integrare $i$ tempi di apprendimento del neofita con i tempi di lavoro e le priorità lavorative.

È intuitivo come la fase di inserimento del nuovo assunto non sia circoscritta al solo periodo di affiancamento del mentore, ma continui con un affidamento graduale di maggiori responsabilità e con l'esecuzione di procedure analitiche sempre più complesse. L'intero iter formativo per il TSLB nell'ambito della batteriologia di base può variare dai 18 ai 24 mesi ed è caratterizzato da una forma di tutoring che è sempre necessaria ad ogni tappa formativa, ma con "un'intensità" che sfuma nel tempo.

Uno strumento indispensabile nel processo di tutorship è il "piano di inserimento lavorativo" in cui vengono individuate le attività e gli strumenti più adeguati al raggiungimento degli obiettivi, secondo un principio di gradualità, i tempi e gli spazi di riflessione, e i criteri di valutazione delle competenze raggiunte. Lo stesso piano può essere usato successivamente dal dipendente per l'autovalutazione e come riferimento nei colloqui periodici col mentore.

Le attività e le modalità attuative devono assicurare un coinvolgimento graduale che porti ad una costruzione organica di competenze, in un arco di tempo che tenga conto della complessità e della ricaduta sull'organizzazione del settore stesso. Molte sono le figure coinvolte nella fase di progettazione e programmazione del percorso di inserimento di un TSLB neoassunto (tecnici, coordinatore tecnico, biologi e medici) che concorrono a definire gli obiettivi formativi specifici, le attività e gli strumenti adeguati a raggiungerli. La scelta del tecnico esperto, con preparazione nel campo della tutorialità, che affiancherà il nuovo assunto come mentore, viene concordata all'interno dell'equipe dell'UO. Egli sarà la persona coinvolta in maggior misura nella tutorship, ma non l'unica, perché anche i dirigenti responsabili dei settori operativi, assieme al coordinatore tecnico ed ai tecnici del servizio instaureranno e condivideranno una relazione di tipo tutoriale. Il coordinatore tecnico si configura come coach e come counsellor. Il counselling sarà attuato anche dai colleghi tecnici. I dirigenti responsabili svolgono un'attività di coaching attraverso la pianificazione e la sperimentazione di attività concrete. Nella fase che precede l'inserimento nell'ambiente di lavoro, il neoassunto incontra il Direttore dell'UO, il laureato responsabile del settore e il coordinatore tecnico: quest'ultimo è il responsabile formale dell'inserimento sia per l'organizzazione che per il dipendente, responsabilità che si concretizzerà nel giudizio di conferma o meno in ruolo. In questo senso, risulta importante il colloquio che il coordinatore tecnico avrà con il neoassunto per approfondirne le eventuali esperienze professionali, aspettative ed esigenze personali. Nella prima giornata il Coordinatore Tecnico presenta al neoassunto il laboratorio, la sua mission, gli aspetti logistici, organizzativi e dei processi lavorativi, illustra le regole formali, i ruoli e le gerarchie del contesto, trasmette il senso della 
cultura organizzativa e professionale che rappresenta il patrimonio consolidato del servizio. Lo accompagna in un giro di presentazione del personale dell'UO, gli consegna i dispositivi di protezione individuale (DPI) e fornisce, per un approfondimento, le normative sulla privacy $e$ sulla sicurezza; infine, presenta il collega che lo affiancherà come mentore.

Il mentore affronta inizialmente con il nuovo assunto la fase di analisi dei bisogni, dove vengono considerate le necessità formative. Questo primo incontro serve al mentore per conoscere il neoassunto e farsi conoscere, ma anche per definire insieme al professionista una sorta di "contratto" contenente le fasi dell'inserimento nel contesto organizzativo e nel settore specifico, le finalità, gli obiettivi, i tempi e le modalità di supervisione. In questo momento risulta indispensabile definire gli obiettivi che il mentore dovrà presidiare (inserimento, supporto emotivo, aspettative).

La negoziazione del contratto si concretizza nella stesura del piano di inserimento condiviso per avviare concretamente il percorso formativo.

Di seguito vengono proposti alcuni obiettivi specifici dell'inserimento del TSLB nel laboratorio di microbiologia. La scelta deriva dall'analisi del profilo professionale del TSLB D.M. 26.09.1994 n. 745 , che delinea gli ambiti delle sue competenze e le responsabilità. Gli obiettivi descritti di seguito contengono le azioni che il TSLB deve essere in grado di compiere per poter raggiungere il risultato di competenza atteso ed i tempi necessari per raggiungere l'abilità richiesta.

La descrizione dettagliata degli obiettivi, delle azioni e dei tempi rappresenta per il tecnico nuovo assunto una valida opportunità per conoscere fin da subito le competenze attese all'interno dell'organizzazione e contribuisce a sviluppare capacità di responsabilizzazione sul proprio percorso di inserimento e di autovalutazione delle performance.

\section{Essere in grado di identificare correttamente $i$ campioni e rilevarne le non conformità (NC)} Azioni:

- Riconoscere specificità operative sulla modalità di prelievo, conservazione e trasporto dei materiali da sottoporre a procedure analitiche microbiologiche attraverso la consultazione del manuale degli esami in uso nel servizio.

- Riconoscere peculiarità sulle modalità di individuazione dei diversi campioni biologici attraverso la consultazione del manuale del sistema informatico e il confronto con il mentor.

- Assegnare e smistare correttamente i campioni nei diversi posti di lavoro.

- Gestire le NC: identificazione, trattamento e registrazione su appositi moduli.

Risultato atteso:

Individuazione precisa dei materiali da sottoporre ad indagine batteriologica nel posto di lavoro assegnato, risolvendo i problemi di identificazione e di idoneità del campione.

Tempo previsto:

due mesi.

2. Essere in grado di applicare le procedure secondo le istruzioni operative (IO) previste nel settore operativo.

Azioni:

- Identificare specificità procedure attraverso la consultazione delle IO per ogni materiale biologico.

- Utilizzare le IO come check-list per seguire e riflettere sulle procedure inizialmente mostrate dal mentore, poi come schema di autovalutazione del proprio operato.

- Pianificare l'attività quotidiana e gestire le priorità nel flusso di lavoro.

- Utilizzare il feed-back del mentore per riposizionare le performance.

\section{Risultato atteso:}

Svolgere in autonomia tecnico-professionale e con cura della qualità delle abilità manuali la propria prestazione lavorativa in diretta collaborazione con il personale dirigenziale preposto alle diverse responsabilità operative di appartenenza. Assumere la responsabilità del corretto adempimento delle procedure analitiche e del proprio operato, nell'ambito delle istruzioni ricevute e delle funzioni affidategli, dell'applicazione dei protocolli di lavoro.

Adeguarsi ai diversi ritmi e carichi di lavoro e dimostrare capacità operativa nell'ottimizzare i flussi di lavoro e pianificare l'attività quotidiana.

\section{Tempo previsto:}

sei mesi, nei quali dovrà sperimentarsi in 2-3 diversi settori operativi a complessità crescente.

3. Applicare le procedure in uso nel servizio relative alla movimentazione, conservazione e uso di reattivi e materiale di consumo

Azioni:

- Utilizzare reattivi e materiale di consumo ponendo attenzione al numero di lotto ed alle scadenze, attenendosi a quanto previsto dalle IO o quanto indicato dal fornitore.

- Rilevare e segnalare al RS le NC dei reattivi e del materiale di consumo.

- Gestire il controllo interno di qualità (CQI) e della Verifica esterna di qualità (VEQ).

- Conoscere la documentazione tecnica specifica. Risultato atteso:

Controllo e verifica del corretto funzionamen- 
to dei reattivi utilizzati e della qualità dei dati; gestione corretta delle risorse materiali date in affidamento.

Tempo previsto: due mesi.

4. Applicare e gestire correttamente le procedure d'uso degli strumenti.

Azioni:

- Applicare correttamente le procedure secondo le IO.

- Verificare la qualità dei dati.

- Provvedere alla manutenzione ordinaria degli strumenti.

- Applicare le procedure di sicurezza.

- Partecipare ai corsi di formazione e/o addestramento su nuove metodologie e nuove tecnologie.

Risultato atteso:

Controllo e verifica del corretto funzionamento delle apparecchiature utilizzate e della loro manutenzione secondo le IO specifiche.

Tempo previsto:

un mese dall'inizio dell'uso della strumentazione.

5. Applicare le norme di sicurezza secondo le direttive Aziendali e le normative vigenti in materia in base alla classificazione di livello di rischio di infettività.

Azioni:

- Conoscere e applicare il manuale della sicurezza e prevenzione.

- Riconoscere le diverse tipologie di rischio nell'ambito del proprio contesto lavorativo.

- Utilizzare in modo appropriato i dispositivi di sicurezza forniti a livello Aziendale per garantire la sicurezza propria ed altrui.

- Attenersi alle precise norme comportamentali in merito al consumo di cibo, al fumo.

- Attenersi alle precise norme Aziendali per lo smaltimento dei rifiuti e in caso di incidente.

- Mantenere in ordine e pulito il proprio posto di lavoro.

Risultato atteso:

Assumere un corretto comportamento atto a garantire la propria ed altrui sicurezza.

Tempo previsto: un mese.

6. Attuare procedure per il passaggio di consegne

Azioni:

- Comunicare con chiarezza verbale e/o scritta i messaggi essenziali.

- Lasciare precisa traccia scritta di tutto ciò che può risultare necessario al corretto svolgimento del lavoro di chi subentra.

- Assistere a passaggi di consegna fra colleghi.

- Effettuare passaggi di consegna sotto supervi- sione.

- Dimostrare capacità di integrazione con i colleghi.

Risultato atteso:

Instaurare un rapporto collaborativo con i colleghi e dimostrare capacità di acquisire e fornire informazioni con chiarezza, con un'ampia ricaduta in termini di qualità sull'intero processo lavorativo nonché di attendibilità del dato analitico stesso.

Tempo previsto:

tre mesi.

7. Essere preciso nell'utilizzo del sistema informatico per l'inserimento del campione nel processo analitico e dei risultati finali.

Azioni:

- Riconoscere nei manuali di istruzione le procedure specifiche di utilizzo del sistema informatico nelle diverse fasi operative.

- Collaborare sia con il personale di segreteria preposto all'accettazione delle richieste di prestazioni degli esami, sia con il personale incaricato allo smistamento dei campioni biologici.

- Verificare la corrispondenza dei campioni con i fogli di lavoro.

- Registrare e inserire con precisione nel sistema informatico i risultati dai fogli di lavoro.

- Archiviare i fogli di lavoro.

Risultato atteso:

Utilizzare correttamente e con precisione il sistema informatico per la gestione del campione nella fase pre-analitica, analitica e postanalitica.

Tempo previsto:

tre settimane.

8. Riconoscere regole formali, ruoli, funzioni, storie professionali del laboratorio e caratteristiche del clima organizzativo.

Azioni:

- Rispettare l'orario di lavoro.

- Comunicare telefonicamente ed in tempo utile ritardi e assenze.

- Dimostrare correttezza nei rapporti con i colleghi e con le altre figure professionali dell'UO.

- Lavorare, stabilendo un clima positivo che favorisca la cooperazione.

- Prevenire i conflitti ricercando la condivisione di regole e valori di comportamento.

- Comunicare, con modalità opportune, eventuali divergenze o critiche alle figure coinvolte.

- Attuare una corretta comunicazione con i reparti aderendo alle indicazioni impartite dai dirigenti.

Risultato atteso:

Cogliere climi e storie del gruppo di lavoro, con rispetto della gerarchia e delle interdipendenze. 


\section{Tempo previsto:} due mesi.

L'elencazione di questa serie di obiettivi specifici, sicuramente non esaustiva, può tuttavia aiutare sia il mentore a sviluppare la sua capacità di formatore, sia il neoassunto ad esprimere una autovalutazione critica.

Per la valutazione delle competenze si utilizza di norma in letteratura (6) una scala di 4 livelli, modificata come segue, considerando che nella fase di inserimento non è ipotizzabile il raggiungimento della competenza esperta:

- completa padronanza: il neoassunto tiene sotto controllo tutte le fasi di un processo lavorativo

- incompleta padronanza: il neoassunto non presidia tutti gli aspetti coinvolti o non sempre

- in fase di sviluppo: il neoassunto evidenzia capacità evolutive ma richiede allenamento nelle diverse attività.

La corrispondenza tra risultato atteso e reale di ogni singolo obiettivo concorrerà alla valutazione formativa del neoassunto.

\section{Il ruolo del mentore nella supervisione del per- corso di inserimento}

Nell'ambito delle metodologie che hanno la finalità di facilitare l'inserimento del neoassunto, la riflessione e la rielaborazione della sua esperienza, hanno particolare importanza, oltre al contratto, il briefing, la pratica e il debriefing.

Le sedute di briefing e debriefing, che rispettivamente precedono e seguono la pratica, sono metodologie di cui si avvale il mentore per trasformare l'esperienza del nuovo assunto in apprendimento.

Le sessioni di briefing hanno lo scopo di:

a) preparare il neofita alla pratica con la trasmissione di informazioni e direttive per l'apprendimento del giorno;

b) stabilire le basi per l'apprendimento dall'esperienza;

c) fargli riconoscere il raggio d'azione ed i limiti del proprio ruolo;

d) stimolare la risoluzione di problemi, l'applicazione delle conoscenze, l'utilizzo del giudizio.

Le sessioni di briefing sono svolte, in un primo periodo, quotidianamente, poi la frequenza sarà determinata dal livello delle conoscenze e delle abilità raggiunte.

L'attività pratica, dopo una fase condotta sotto supervisione del tutor, serve per mettere alla prova conoscenze ed abilità, rendere consapevoli dei rischi, migliorare destrezza, precisione e capacità di risoluzione dei problemi, interpretare la teoria nel suo legame con la pratica.

Il debriefing è l'analisi dell'esperienza per attuare la trasformazione dell'esperienza stessa in apprendimento. Esso non è una fase conclusiva, ma conduce ad una nuova esperienza, secondo il ciclo briefing - pratica - debriefing, ritarata in base al feedback ricevuto. Il debriefing può rappresentare non solo una verifica del livello della performance del nuovo assunto, ma anche di quella del mentore. Egli infatti può essere stimolato dal feedback del novizio a porsi dei quesiti rivolti alla propria autovalutazione. Con frequenza quindicinale, le sessioni di debriefing sono dedicate alla valutazione del percorso fatto utilizzando come strumento il piano di inserimento.

\section{CONCLUSIONI}

Elementi chiave per la formazione e la generazione di nuova conoscenza sono l'esperienza e la riflessione critica sulla pratica professionale. La creazione di professionisti esperti all'interno delle organizzazioni viene facilitato da figure con competenze pedagogiche nell'ambito della tutorialità. E possibile ipotizzare, attorno al nuovo assunto nel laboratorio di Microbiologia, un setting tutoriale ed una assunzione di funzioni tutoriali, che di volta in volta possono declinarsi con modalità ed obiettivi diversi.

La condivisione del progetto di inserimento concorre a promuovere una cultura della tutorship all'interno dell'organizzazione ed un apprendimento finalizzato allo sviluppo delle potenzialità insite tanto nell'individuo che nell'organizzazione stessa.

La tutorship all'interno del Laboratorio di Microbiologia non si deve configurare come un processo informale e spontaneo: va progettato, promosso ed implementato:

a) un programma formale di tutorship;

b) un percorso formativo per sviluppare le competenze pedagogiche richieste ai formatori.

Questa progettualità ha bisogno di essere sostenuta con una leadership che si caratterizzi come Manager Educante.

\section{BIBLIOGRAFIA}

1. Barrows HS. Il processo tutoriale. Smith Kline, 1988 (ed. italiana a cura di Martin A)

2. Dewey J. Experience and Education. New York: Macmillan,1938.

3. Mongarli L, et al. La valutazione del neoassunto Tecnico Sanitario di Laboratorio Biomedico. Microb. Med 1999, 14(1): 41-52.

4. Quaglino GP. Scritti di formazione 1978-1998. Franco Angeli Ed. Milano 1999; 228-35.

5. Saiani L. Il tirocinio nelle professioni socio-sanitarie ed educative. In: Viaggi guidati: Il tirocinio e il processo tutoriale nelle professioni sociali e sanitarie, Franco Angeli Ed., Milano 1997, 61-82.

6. Saiani L, Palese A. Guida per pianificare lo sviluppo 
professionale e documentare le competenze. Assistenza infermieristica e ricerca, 2002; 2 (21).

7. Sarchielli G. Evoluzione delle professioni e significato del tirocinio. In: Castellucci A, Saiani L, Sarchielli G, Marletta L. Viaggi guidati - Il tirocinio nelle professioni sociali e sanitarie, Franco Angeli Ed.,Milano, 1997, 35-51

8. Sarchielli G. Le abilità di base e il ruolo attivo del soggetto nella costruzione di risposte professionali competenti. In Risorsa Uomo, 1996.

9. Visconti A. Microbiologia clinica: presente e futuro. Notizie AMCLI 2002, 5 (5): 7-10

Lavoro estratto dalla tesi del Master in Modelli e metodi della tutorship nella formazione degli operatori della salute - Università degli Studi di Verona - Facoltà di Medicina e Chirurgia presentata da Iole Caola nell'Anno Accademico 2001-2002

\section{Iole Caola}

Laboratorio di Microbiologia e Virologia

Ospedale S.Chiara - 38100 Trento

Tel.: 0461903270 - Fax 0461903615

E-mail: iole.caola@apss.tn.it 